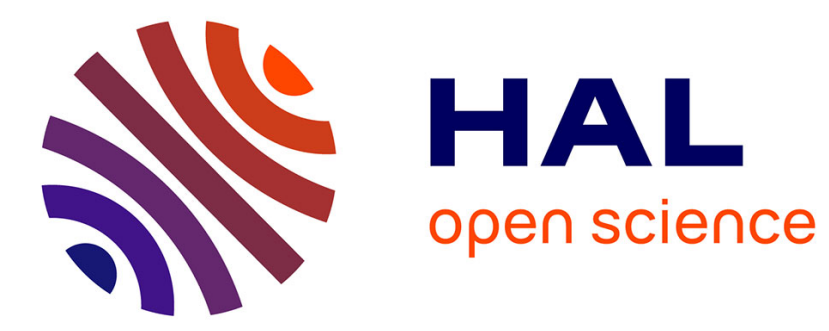

\title{
On nonlinear dynamics of planar shear indeformable beams
}

\author{
Angelo Luongo, Giuseppe Rega, Fabrizio Vestroni
}

\section{To cite this version:}

Angelo Luongo, Giuseppe Rega, Fabrizio Vestroni. On nonlinear dynamics of planar shear indeformable beams. Journal of Applied Mechanics, 1986, 53 (31-32), pp.619-624. hal-00789126

\section{HAL Id: hal-00789126 \\ https://hal.science/hal-00789126}

Submitted on 15 Feb 2013

HAL is a multi-disciplinary open access archive for the deposit and dissemination of scientific research documents, whether they are published or not. The documents may come from teaching and research institutions in France or abroad, or from public or private research centers.
L'archive ouverte pluridisciplinaire HAL, est destinée au dépôt et à la diffusion de documents scientifiques de niveau recherche, publiés ou non, émanant des établissements d'enseignement et de recherche français ou étrangers, des laboratoires publics ou privés. 
A. Luongo

Istituto di Scienza delle Costruzioni, University of Rome, Roma, Italy 00184

\section{G. Rega}

\section{F. Vestroni}

Istituto di Scienza delle Costruzioni, University of L'Aquila, L'Aquila, Italy 67100

\section{On Nonlinear Dynamics of Planar Shear Indeformable Beams}

The planar forced oscillations of shear indeformable beams with either movable or immovable supports are studied through a unified approach. An exact nonlinear beam model is referred to and a consistent procedure up to order three nonlinearities is followed. By eliminating the longitudinal displacement component through a constraint condition and assuming one mode, the problem is reduced to one nonlinear differential equation. A perturbational solution in the neighborhood of the resonant frequency is determined and the stability of the steady-state solutions is studied. The dependence of the phenomenon on the geometrical and mechanical characteristics of the system is put into light and the frequency-response curves for different boundary conditions are furnished.

\section{Introduction}

The planar nonlinear vibrations of shear indeformable beams is the subject of several works in the literature. Most of them are devoted to the study of an axially restrained simplysupported beam taking into account the most important nonlinearities due to the stretching of the beam axis only (Wojnowsky Krieger, 1950; Eisley, 1964; Evensen, 1968; Ray and Bert, 1969; Mei, 1973; Nayfeh et al., 1974; Bhashyam and Prathap, 1980). A few have dealt with the oscillations of beams with no axial restraints (Wagner, 1965; Atluri, 1973); in this case the effects of large curvature and longitudinal inertia are included, as followed in some recent works on nonplanar motions as well (Crespo da Silva and Glynn, 1978; Hyer, 1980; Luongo et al., 1984a).

In this work a unified approach is followed to study the planar forced vibrations of beams with or without axial constraints, by using an exact continuum model of shear indeformable beam. An internal constraint, different in the two cases, is introduced for eliminating systematically the longitudinal displacement and the eigenfunction of the linearized system is referred to for reducing the problem to one nonlinear ordinary differential equation. The solution is pursued through the multiple time scale perturbation method by retaining terms up to the second order of the perturbation parameter. The main quantities which govern the phenomenon in the two cases considered are pointed out. The frequency-response curves are evaluated for different boundary conditions and the stability of stationary oscillations is studied. More detailed analytical developments and results of the problem presented here are reported elsewhere (Luongo et al., 1984b).

\section{Equations of Motion}

A uniform initially straight beam supported in an arbitrary manner is considered. Let $\ell$ be the undeformed length of the beam and $m$ the mass per unit length. In the planar motion the deformed shape of the beam is described by an elastic displacement of its centroid $C$, with longitudinal and transverse components $u(s, t)$ and $v(s, t)$, and by atation of the beam cross section about $C$ of amplitude $\theta(s, t)$.

The condition of zero-transverse-shear furnishes an explicit relation between $\theta$ and $u, v$ :

$$
\tan \theta=v^{\prime} /\left(1+u^{\prime}\right)
$$

where the prime denotes partial differentiation with respect to arc-length $s$. The curvature

$$
\mu(s, t)=\theta^{\prime}(s, t)
$$

is assumed as a measure of the flexural deformation and elongation rate

$$
\epsilon(s, t)=\left[\left(1+u^{\prime}\right)^{2}+v^{\prime 2}\right]^{1 / 2}-1
$$

as a measure of the axial deformation. Referring to small elastic strains, the elastic potential energy $\Pi(\epsilon, \mu)$ and the kinetic energy $K(\dot{u}, \dot{v})$ of the beam are quadratic diagonal forms of the deformation field and of the velocity field, respectively; in the latter the rotary inertia is neglected since it gives very little contribution if nonstructural mass is not present, as it can be deduced from the solution given by Atluri (1973). The dot means differentiation with respect to time. In view of examining the nonlinear oscillations with order-three nonlinearities, the relations (1), (2), and (3) are consistently expanded in Taylor series.

The physics of the problem considered suggests the introduction of simplifying assumptions concerning the axial deformation. For the beam with a movable end the axial deformation is negligible and the restriction of the elastica - no extension of the neutral axis - is used. For the beam whose ends are both restrained from axial displacement, the inertia term is neglected; so, according to the longitudinal equilibrium equation (equation (B.2) in Appendix B), the axial force can be assumed constant along the beam, coherently 
with the order of approximation adopted. For both boundary conditions, movable and immovable ends, the axial deformation is thus a function of time only: $\epsilon(s, t)=e(t)$.

Taking into account this circumstance, $u^{\prime}=0\left(u^{\prime 2}\right)$ results and retaining terms up to third order, equation (3) leads to:

$$
u^{\prime}=e(t)-\frac{1}{2} v^{\prime 2}
$$

which allows one to express the displacement $u$ in terms of the transverse component:

$$
u(s, t)=u(0, t)+e(t) s-\int_{0}^{s} \frac{1}{2} v^{\prime 2} d s
$$

Considering the case of the fixed support at 0 , when the end $L$ is free in the $u$ direction, $e(t) \equiv 0$ and

$$
u(s, t)=-\int_{0}^{s} \frac{1}{2} v^{\prime 2} d s,
$$

when the end $L$ is fixed, $u(\ell, t)=0$ and

$$
u(s, t)=\frac{s}{\ell} \int_{0}^{\ell} \frac{1}{2} v^{\prime 2} d s-\int_{0}^{s} \frac{1}{2} v^{\prime 2} d s
$$

In order to obtain a nondimensional ordinary equation of motion, the following normalized variables are introduced:

$$
\begin{gathered}
\tilde{s}=s / \ell \quad \bar{t}=\omega t \\
\tilde{v}(s, t)=v(s, t) / \ell=\phi(s) q(t) \\
\tilde{p}(s, t)=p(s, t) \ell^{\beta} / E I=\psi(s) P \cos \Omega t
\end{gathered}
$$

where $\omega$ is the linear frequency of the oscillation under study, the displacement $v(s, t)$ is described by one mode and an assigned spatial distribution is assumed for the transverse load $p(s, t)$ per unit length of beam, harmonic of frequency $\Omega$.

A) Movable End. Taking into account equation (6), the Hamiltonian reads:

$$
\begin{gathered}
H(v)=\int_{t_{1}}^{t_{2}} \int_{0}^{\ell}\left\{\frac{1}{2} m\left[\left(\int_{0}^{s} \frac{1}{2}\left(v^{\prime 2}\right)^{\bullet} d s\right)^{2}+\dot{v}^{2}\right]\right. \\
\left.-\frac{1}{2} E I\left(v^{\prime \prime 2}+v^{\prime 2} v^{\prime \prime 2}\right)+p v\right\} d s d t
\end{gathered}
$$

After introducing the nondimensionalized quantities, via equations (8)-(11), it depends on $q$ only. The stationary condition leads to the ordinary differential equation of motion:

$$
\ddot{q}+q+b q^{3}+c\left(q \dot{q}^{2}+q^{2} \ddot{q}\right)=p_{0} \cos \Omega t
$$

where the dot now means derivative with respect to the nondimensional time $\tilde{t}$ which makes the coefficients of the linear terms equal to unity after divided for $\omega^{2}$, being

$$
\omega^{2}=\frac{E I}{m \ell^{4}} \frac{h_{2}}{h_{1}}
$$

Besides the coefficients of the other terms in equation (13) follow:

$$
b=2 \frac{h_{3}}{h_{2}} \quad c=\frac{h_{4}}{h_{1}} \quad p_{0}=P \frac{h_{0}}{h_{2}}
$$

where $h_{i}$ are dimensionless quantities dependent on the shape functions $\phi(s)$ and $\psi(s)$ and they are defined in Appendix A.

B) Immovable End. The Hamiltonian which governs this case reads:

$$
\begin{gathered}
H(v)=\int_{t_{1}}^{t_{2}} \int_{0}^{\ell}\left\{\frac{1}{2} m \dot{v}^{2}-\frac{1}{2} E I\left(v^{\prime \prime 2}+v^{\prime 2} v^{\prime 2}\right.\right. \\
\left.-\frac{1}{\ell} v^{\prime \prime 2} \int_{0}^{\ell} v^{\prime 2} d s\right)-\frac{1}{8} \frac{E A}{\ell^{2}}\left(\int_{0}^{\ell} v^{\prime 2} d s\right)^{2} \\
+p v\} d s d t
\end{gathered}
$$

where the kinetic energy associated with the longitudinal component has been omitted according to the assumption that the longitudinal inertia forces are negligible. An equation of motion similar to equation (13) is obtained with the elastic nonlinear term only $(c=0)$, being

$$
b=\frac{1}{h_{2}}\left[\frac{1}{2} \frac{E A \ell^{2}}{E I} h_{4}^{2}+2\left(h_{3}-h_{2} h_{4}\right)\right]
$$

It thus follows that the two cases of beam with movable and immovable ends exhibit nonlinearities of cubic order only because of symmetry of the continuum problem, but they are elastic only in the latter case while inertia nonlinearities also are present in the former one. Furthermore the analysis of the coefficients of the motion equations puts into light that, in terms of the nondimensionalized parameters adopted, the nonlinear phenomenon is completely independent of the geometrical and mechanical properties of the beam in case A); while, in case B), the dependence of these properties is controlled by the slenderness parameter $\lambda^{2}=E A \ell^{2} / E I$. This parameter has generally large values and makes the first term due to stretching predominant compared to others associated with the nonlinear curvature.

\section{Perturbation Solution}

In the following the solution to the equation of motion is obtained by referring to the general equation (13) which contains both elastic and inertia nonlinear terms. The case of primary resonance, that is the frequency of excitation near a natural frequency of the beam, is studied.

Let $\bar{q}$ and $\bar{p}_{0}$ be two new variables of order $0(1)$. Since the beam is being excited near resonance, the response would be quite large, even with small values of $p_{0}$; so, if $\epsilon$ is a small but finite parameter, it can be stated:

$$
q=\epsilon^{1 / 2} \bar{q} \quad p_{0}=\epsilon^{3 / 2} \bar{p}_{0}
$$

in fact, bearing in mind the nondimensionalization (11) of $p$, this ordering is suitable for the study of oscillations of amplitude considerably greater than the static deflection. Substitution of equation (20) into equation (13) yields:

$$
\ddot{q}+q=-\epsilon b q^{3}-\epsilon c\left(q \dot{q}^{2}+q^{2} \ddot{q}\right)+\epsilon p_{0} \cos \Omega t
$$

where the overbar is omitted.

To obtain an approximate solution for the maximum amplitude and the period of the resonant oscillation the method of multiple time scales is adopted (Nayfeh and Mook, 1979), according to which the time scale $t$ is replaced by new independent time variables $T_{n}=\epsilon^{n} t$. The solution is expanded in power series of $\epsilon$ as:

$$
\begin{array}{r}
q(t ; \epsilon)=q_{0}\left(T_{0}, T_{1}, T_{2}\right)+\epsilon q_{1}\left(T_{0}, T_{1}, T_{2}\right) \\
+\epsilon^{2} q_{2}\left(T_{0}, T_{1}, T_{2}\right)
\end{array}
$$

and the frequency of excitation is expressed as:

$$
\Omega=1+\epsilon \sigma_{1}+\epsilon^{2} \sigma_{2}
$$

where $\sigma_{n}=0(1)$. Substituting equation (22) into equation (21), expanding the ordinary derivatives in terms of partial derivatives with respect to $T_{n}$ and equating the coefficients of equal powers of $\epsilon$, the following equations are obtained at the orders $\epsilon^{0}, \epsilon$, and $\epsilon^{2}$, respectively:

$$
\begin{gathered}
D_{00} q_{0}+q_{0}=0 \\
D_{00} q_{1}+q_{1}=-2 D_{01} q_{0}-b q_{0}^{3}-c q_{0}\left[\left(D_{0} q_{0}\right)^{2}\right. \\
\left.\quad+q_{0} D_{00} q_{0}\right]+p_{0} \cos \Omega t
\end{gathered}
$$

where $D_{k}=\partial / \partial T_{k}$ and $D_{j k}=\partial^{2} / \partial T_{j} \partial T_{k}$. 
The solution to equation $(24)_{1}$ can be written as

$$
q_{0}=A\left(T_{1}, T_{2}\right) e^{i T_{0}}+\text { complex conjugate }
$$

Introducing the polar form

$$
A\left(T_{1}, T_{2}\right)=\frac{1}{2} a\left(T_{1}, T_{2}\right) \exp \left[i \beta\left(T_{1}, T_{2}\right)\right]
$$

and the new variable

$$
\gamma=\sigma_{1} T_{1}+\sigma_{2} T_{2}-\beta
$$

the condition of vanishing of the secular term in equation $(24)_{2}$ gives, after separation into real and imaginary parts:

$$
\begin{aligned}
a^{\prime} & =\frac{1}{2}-p_{0} \sin \gamma \\
a \gamma^{\prime} & =a \sigma_{1}-\frac{1}{8}(3 b-2 c) a^{3}+\frac{1}{2} p_{0} \cos \gamma
\end{aligned}
$$

where the prime denotes partial differentiation with respect to $T_{1}$.

Solving for $q_{1}$, substituting it into equations $(24)_{2}$, together with equation (25) and by setting to zero the coefficient of the secular term, in which use is made of equations (26)-(28), gives:

$$
\begin{array}{r}
\hat{a}=-\frac{1}{4} p_{0} \sin \gamma\left[\sigma_{1}+\frac{1}{8}(9 b+2 c) a^{2}\right] \\
a \hat{\gamma}=a \sigma_{2}-\frac{1}{4} p_{0} \cos \gamma\left[\sigma_{1}+\frac{3}{8}(b+2 c) a^{2}\right] \\
+\frac{1}{256}\left[15 b^{2}+36 b c-36 c^{2}\right] a^{5}
\end{array}
$$

where ( ${ }^{\wedge}$ ) denotes partial differentiation with respect to $T_{2}$.

The two sets of coupled first-order differential equations (28) and (29) describe the variation of the amplitude and phase of the oscillation on the slow scales $T_{1}$ and $T_{2}$; on the real time $t$ this variation is described by:

$$
\begin{aligned}
& \dot{a}=\epsilon a^{\prime}+\epsilon^{2} \hat{a} \\
& \dot{\gamma}=\epsilon \gamma^{\prime}+\epsilon^{2} \hat{\gamma}
\end{aligned}
$$

Steady-State Motion. In the steady-state motion, amplitude and phase are constant, $a(t)=a_{0}, \gamma(t)=\gamma_{0}$ and they are determined by imposing the vanishing of the right terms of the system (30). Since it must be valid for any value of $\epsilon$, the two conditions $a^{\prime}=\gamma^{\prime}=0$ and $\hat{a}=\hat{\gamma}=0$ follow. From the first ones $\gamma_{0}$ and $\sigma_{1}$ are determined; $\sigma_{2}$ is obtained from the second ones.

Substituting $\sigma_{1}$ and $\sigma_{2}$ into equation (23) and eliminating the parameter $\epsilon$, coming back according to equation (20), a frequency-response equation for an assigned intensity $p_{0}$ of the force is obtained:

$$
\begin{gathered}
\Omega=1+\left(\frac{3}{8} b-\frac{c}{4}\right) a_{0}^{2}+\left[-\frac{15}{256} b^{2}+\frac{9}{64} c(c-b)\right] a_{0}^{4} \\
\mp \frac{p_{0}}{2 a_{0}} \pm\left(\frac{3}{16} b+\frac{1}{8} c\right) p_{0} a_{0}-\frac{p_{0}^{2}}{8 a_{0}^{2}}
\end{gathered}
$$

The steady-state solution at the $\epsilon^{2}$-order reads:

$q(t)=a_{0} \cos \left(\Omega t-\gamma_{0}\right)+\frac{1}{32}(b-2 c) a_{0}^{3} \cos 3\left(\Omega t-\gamma_{0}\right)$

The steady-state resonant motion exhibits the same frequency of the excitation with a phase shifted from that of the excitation by $\left(-\gamma_{0}\right)$ and at this order it is not simply sinusoidal for the presence of the harmonic with frequency $3 \Omega$. When there is no excitation, equation (31) describes the relation between the frequency and the amplitude of the free oscillation of the beam; the temporal law of the motion is given again by equation (32). The double sign of loading terms of equation
(31) produces a multivalued function which actually modifies strongly the general features of the response of the system, for which the occurrence of a stable oscillation is governed by the initial conditions.

Stability of the Steady-State Solutions. For a given excitation $p_{0}$, the stability of the different solutions lying along the response curve can be determined by analyzing the nature of the singular points of equations (30). To this scope a perturbation of $a$ and $\gamma$ is introduced by writing $a=a_{0}+a_{1}$ and $\gamma=\gamma_{0}+\gamma_{1}$ and linearizing equations (30) in $a_{1}$ and $\gamma_{1}$ the following set of first-order differential equations is obtained:

$$
\begin{gathered}
\dot{a}_{1}=g_{1}\left(a_{0}\right) \gamma_{1}=\left\{ \pm \epsilon \frac{p_{0}}{2}+\epsilon^{2} \frac{p_{0}}{8 a_{0}}\left(p_{0} \mp 3 b a_{0}^{3}\right)\right\} \gamma_{1} \\
\dot{\gamma}_{1}=g_{2}\left(a_{0}\right) a_{1}=\left\{-\epsilon\left[\frac{1}{4}(3 b-2 c) a_{0} \pm \frac{1}{2} \frac{p_{0}}{a_{0}^{2}}\right]\right. \\
+\epsilon^{2}\left[\frac{1}{64}\left(15 b^{2}+36 b c-36 c^{2}\right) a_{0}^{3}-\frac{1}{8} \frac{p_{0}^{2}}{a_{0}^{3}}\right. \\
\left.\left.\mp \frac{1}{4} p_{0} c\right]\right\} a_{1}
\end{gathered}
$$

The stability of the oscillations depends on the eigenvalues of the coefficient matrix of system (33); the steady-state motion is unstable when $g_{1}\left(a_{0}\right) g_{2}\left(a_{0}\right)>0$ and the locus of points which separates stable and unstable solutions is determined by the condition:

$$
g_{1}\left(a_{0}\right) g_{2}\left(a_{0}\right)=0 \text {. }
$$

$g_{1}\left(a_{0}\right)$ vanishes when the external force is zero: this is represented on the $\Omega-a_{0}$ plane by the backbone curve. The other condition $g_{2}\left(a_{0}\right)=0$, solved with respect to $p_{0}$ and substituted into equation (49), gives:

$$
\begin{array}{r}
\Omega=1+\epsilon \frac{3}{8}(3 b-2 c) a_{0}^{2}+\epsilon^{2} \frac{1}{256}\left(-147 b^{2}\right. \\
\left.+276 c^{2}-276 b c\right) a_{0}^{4}
\end{array}
$$

which defines together with the backbone curve the region of unstable oscillations.

\section{Numerical Results}

Equation (31) is used to draw the frequency-response curve of the forced motion together with the backbone curve of the free motion for the two different axial boundary conditions which are studied separately.

Immovable End. Past works have shown that the frequency-response curve is completely independent of the geometrical and mechanical properties of the beam when the amplitude of oscillation is nondimensionalized with respect to the inertia radius $\rho$. This behavior occurs where the nonlinearities due to the stretching of the beam axis only are taken into account: in the present work, where the nonlinear curvature is also considered, this is not true anymore. However, since the more important contribution is that of the axial deformation, as already remarked in the preceding Section, it is still preferable to represent the frequency-response curve assuming a nondimensional amplitude with respect to $\rho$.

By replacing $\tilde{v}=v / \ell$ used in analytical treatment with $\tilde{v}=v / \rho$, the coefficients of the frequency-response curve defined by equations (17) and (19) change as follows:

$p_{0}=P\left(\frac{\ell}{\rho}\right) \frac{h_{0}}{h_{2}} \quad b=\left[\frac{1}{2} h_{4}^{2}+2\left(\frac{\rho}{\ell}\right)^{2}\left(h_{3}-h_{2} h_{4}\right)\right] / h_{2}$

It is seen that, due to the smallness of the parameter $(\rho / \ell)^{2}$, its variation produces little modification in the response. In Fig. 1 the correction of the natural frequency of the first mode versus the amplitude of free oscillation is reported for three 

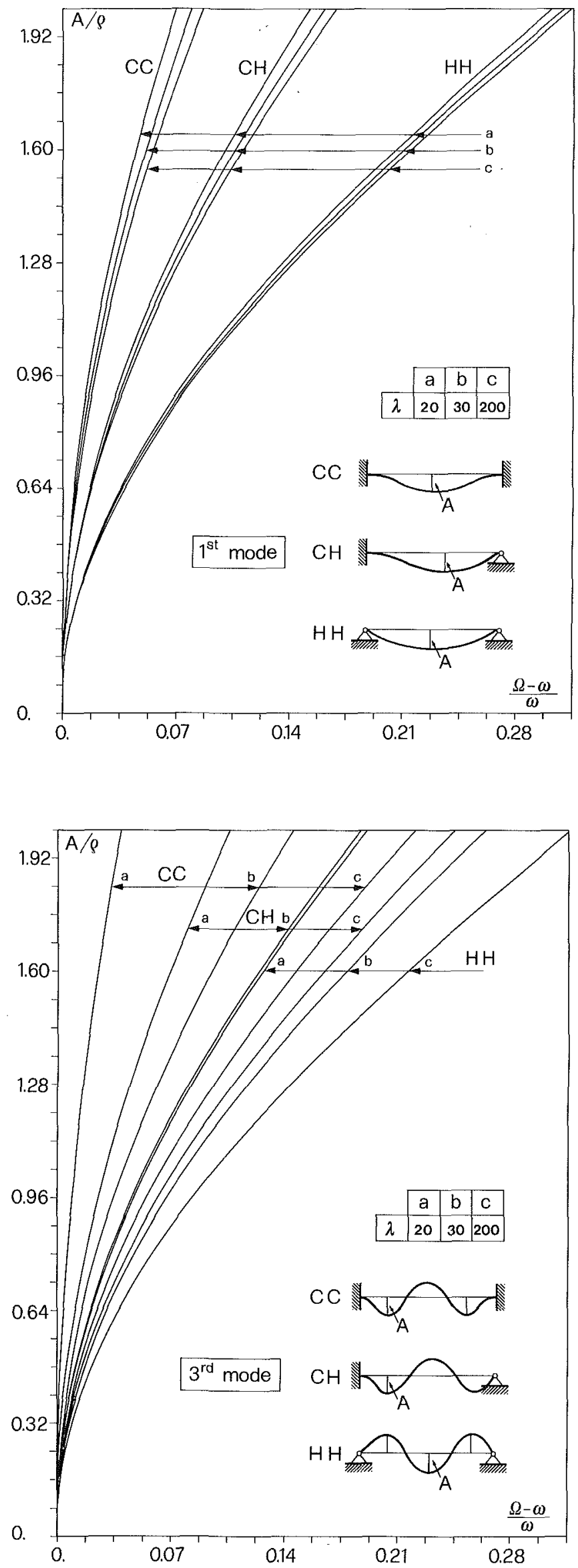

Figs. 1, 2 Frequency-amplitude relationship for different boundary conditions (immovable end)

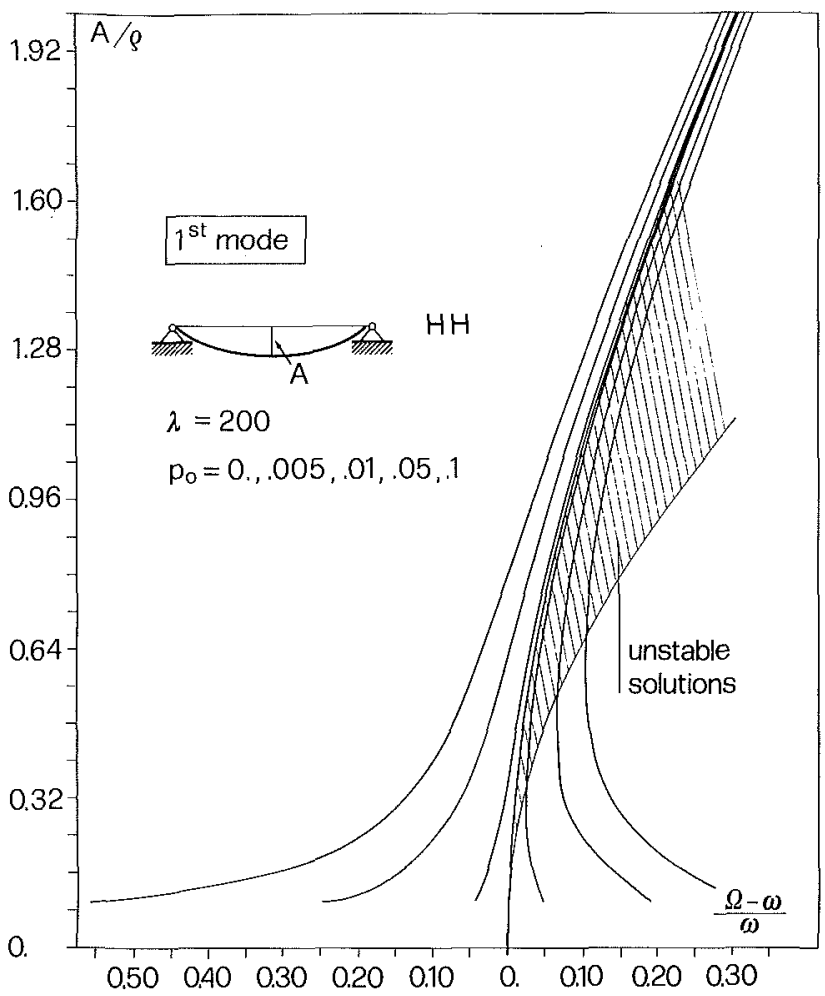

Fig. 3 Frequency-response relationship for hinged-hinged beam

boundary conditions and three different values of the slenderness. The largest corrections are those of hinged-hinged beams for which a deformed configuration of given amplitude is associated with the largest value of stretching. The influence of the parameter $\lambda$ is quite small in all cases considered, greater for clamped-clamped beams since lower corrections of frequency take place in this case but with greater value of curvature with respect to the other two boundary conditions.

The analogous curves for the third mode produce a very different picture (Fig. 2). This is due to both of the following circumstances: for the higher modes the eigenfunctions tend to become more similar, so the differences of the frequencyamplitude curve for the various boundary conditions are less pronounced; besides, these eigenfunctions are characterized by greater values of the curvature, so the effect of the parameter $\lambda$ on the third mode becomes very strong. Therefore, in this case the curves for the three boundary conditions are not separated from each other, as it happens in Fig. 1.

For the first mode the solution obtained for $\lambda>100$ can be considered coincident with the classical solution obtained neglecting nonlinear curvature effects (Wojnowsky Krieger, 1950; Evensen, 1968). In contrast, Fig. 2 shows that those effects cannot be omitted in the analysis of the higher modes.

For the beam with larger nonlinear behavior $(H H)$ oscillating in the first mode, the frequency-response curves are reported in Fig. 3 and the region of unstable steady-state solutions is evidenced on the basis of equation (35).

Movable End. For these boundary conditions the response is completely independent of the mechanical characteristics of the beam when the amplitude is nondimensionalized with respect to length.

In Fig, 4 the frequency-amplitude curves for four different boundary cases are collected. If two corresponding boundary conditions with immovable and movable ends are compared, it can be observed that the frequency corrections are much lower in the latter case for the same oscillation amplitude and 


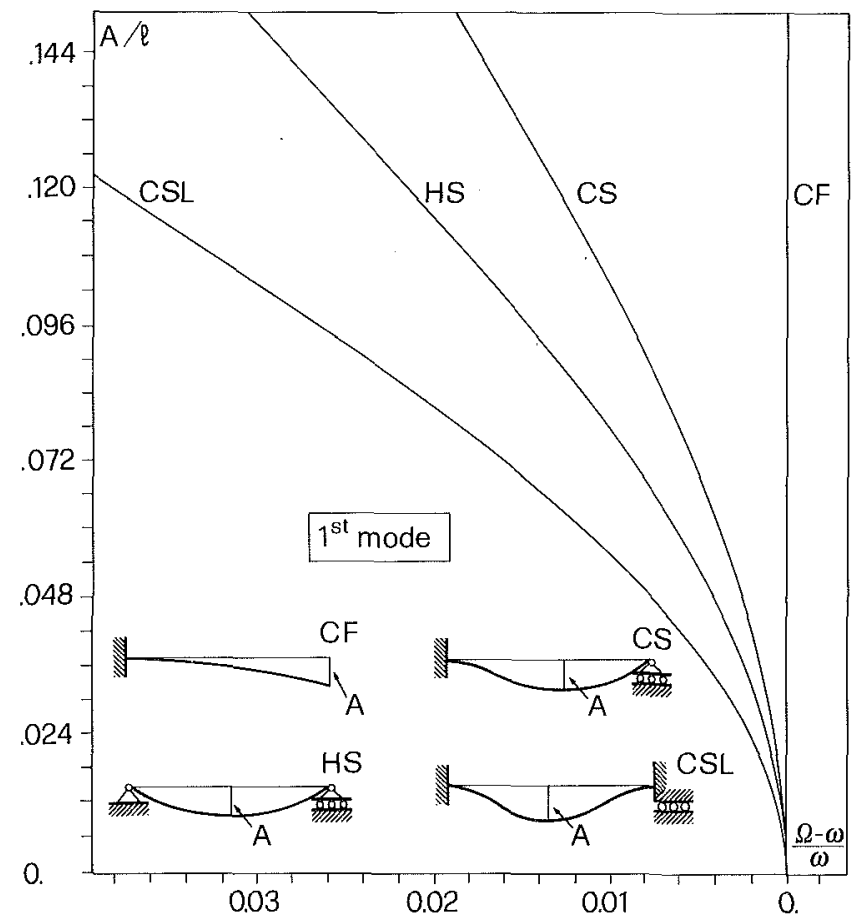

Fig. 4 Frequency-amplitude relationship for different boundary conditions (movable end)

in general, apart from the free-fixed beam, the behavior is of softening type. This can be related to two different reasons: the first, which is more general, is that according to the physics of the problem weak nonlinearities arise in the motion of beams not axially restrained; the second, which is more specific, is that in this case both inertia and elastic nonlinearities are present but they are opposite in sign. On the basis of the second circumstance, the fixed-free beam shows an almost linear behavior, just because the hardening effect of the elastic term is a little higher than the softening effect of intertia terms; this latter term is always prevailing for the other boundary conditions. For the third mode the relevant curves are always softening with higher frequency corrections since in the higher modes the hardening contribution of the elastic term is negligible.

The case of the hinged-supported beam is only studied by Atluri (1973); results are furnished for the first and the third modes. The latter mode is in very good agreement with those obtained herein, while some discrepancy occurs between those for the first mode; this is due to a different evaluation of the nonlinear elastic term the effect of which is important only in the first mode where the frequency corrections are very little. Although this discrepancy does not cause modification in the qualitative behavior and produces high percent differences where the frequency corrections are very low, it is worthy to examine the matter more closely (see Appendix B).

Taking into account that the frequency corrections are little in any case, the frequency-response curves are drawn only for the case of hinged-supported beam in Fig. 5. It can be seen that in this case the low slope of the backbone curve makes the region of unstable solutions very narrow.

\section{Conclusions}

The planar large amplitude forced vibrations of shear indeformable beams are studied for the two different cases of movable and immovable support at one end. An exact nonlinear beam model is referred to and any kind of boundary conditions are considered; a unified approach is followed to

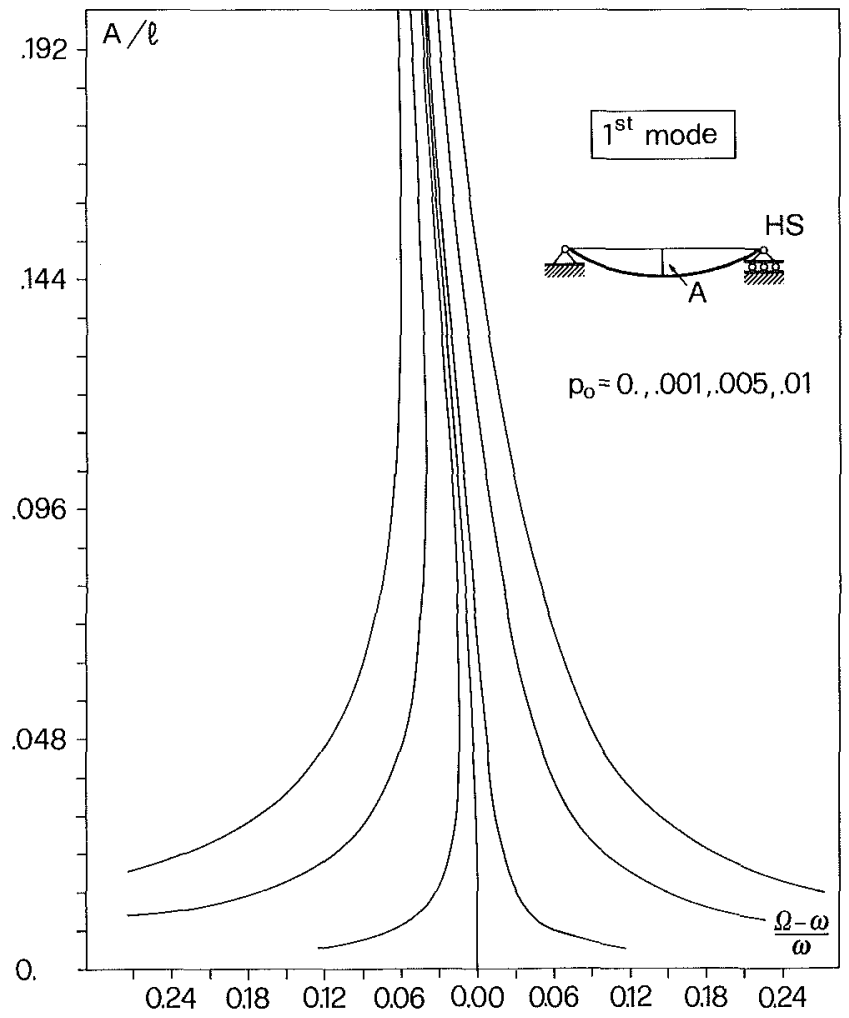

Fig. 5 Frequency-response relationship for hinged-supported beam

tackle an asymptotic consistent solution of the problem. The steady-state motions and their stability are studied.

The oscillatory phenomenon of the beam with the movable end does not depend on the mechanical characteristics when the amplitude is nondimensionalized with respect to the length. For the beam with the immovable end it is convenient to make use of the nondimensionalization with respect to the inertia radius but also in this representation the phenomenon is not completely independent of mechanical characteristics, as it is found in the literature, due to effects of nonlinear curvature; these become quite important for the modes higher than the first.

For the beam which is axially restrained, the effects of nonlinearities, mainly due to the stretching of the beam axis, are more pronounced with respect to the other case where the nonlinear behavior is associated with the elastic and longitudinal inertia terms, so that in the former boundary case the range of frequency in which the frequency-response curve is multivalued is wider, as well as the region of unstable solutions.

\section{Acknowledgment}

This research was partially supported by MPI $60 \%$ funds.

\section{References}

Atluri, S., 1973 " Nonlinear Vibration of Hinged Beam Including Nonlinear Inertia Effects," ASME Journal of Applied Mechanics, Vol. 40, pp. 121-126. Bhashyam, G. R., and Prathap, G., 1980, "Galerkin Finite Element Method for Non-Linear Beam Vibrations," Journal of Sound and Vibration, Vol. 72, pp. 191-203.

Crespo da Silva, M. R. M., and Glynn, C. V. C., 1978, "Non-Linear Flexural-Flexural-Torsional Dynamics of Inextensional Beams I. Equations of Motion," Journal of Structural Mechanics, Vol. 6, pp. 437-448.

Eisley, J. G., 1964, "Nonlinear Vibration of Beams and Rectangular Plates," Zeitschrift für Angewandte Mathematik und Physik, Vol. 15.

Evensen, D. A., 1968, "Nonlinear Vibrations of Bearns with Various Boundary Conditions," American Institute of Aeronautics and Astronautics Journal, Vol. 6, pp. $370-372$.

Hyer, M. W., 1980, "Nonplanar Motions of a Base-Excited Cantilever," In- 
ternational Conference on Recent Advances in Structural Dynamics, Southamp ton, Vol. 2, pp. 621-630.

Luongo, A., Rega, G., and Vestroni, F., 1984a, "Accurate Nonlinear Equations and a Perturbational Solution for the Free Nonplanar Vibrations of Inextensional Beams," 2nd International. Conference on Recent Advances in Structural Dynamics, Southampton, Vol. 1, pp. 341-350.

Luongo, A., Rega, G., and Vestroni, F., 1984b, "Nonlinear Dynamics of Planar Shear-Indeformable Beams," Ist. Scienza delle Costruzioni, L'Aquila, Report No. 80

Mei, C., 1973, "Finite Element Displacentent Method for Large Amplitude Free Flexural Vibrations of Beams and Plates," Computers and Structures, Vol. 3, pp. 163-174.

Nayfeh, A. H., Mook, D. T., and Lobitz, D. W., 1974, "NumericalPerturbation Method for the Nonlinear Analysis of Structural Vibrations," American Institute of Aeronautics and Astronautics Journal, Vol. 12, pp. 1222-1228.

Nayfeh, A. H., and Mook, D. T., 1979, Nonlinear Oscillations, Wiley, New York.

Ray, J. D., and Bert, C. W., 1969, "Non-Linear Vibrations of a Beam With Pinned Ends," ASME Journal of Engineering for Industry, Vol. 91, pp. $977-1004$.

Wagner, H., 1965, "Large-Amplitude Free Vibrations of a Beam," ASME JOURNAL OF APPLIED Mechanics, pp. 887-892.

Wojnowsky Krieger, S., 1950, "The Effect of an Axial Force on the Vibration of Hinged Bars," ASME Journal of Applied Mechanics, Vol. 17, pp. 35-36

\section{A P P E N D I X A}

The coefficients $h_{0}, h_{1}, h_{2}, h_{3}$ are the same for the two different boundary conditions considered and they are defined as follows:

$$
\begin{array}{ll}
h_{0}=\int_{0}^{1} \psi(s) \phi(s) d s & h_{1}=\int_{0}^{1} \phi^{2}(s) d s \\
h_{2}=\int_{0}^{1} \phi^{\prime 2}(s) d s & h_{3}=\int_{0}^{1} \phi^{\prime 2}(s) \phi^{\prime \prime 2}(s) d s
\end{array}
$$

The coefficient $h_{4}$ results:

\section{Case A)}

$h_{4}=\int_{0}^{1}\left(\int_{0}^{s} \phi^{\prime 2}(s) d s\right)^{2} d s$

\section{Case B)}

The prime denotes derivative with respect to $\tilde{s}$, the tilde being dropped for the sake of brevity.

\section{A P P E N D I X B}

The frequency-amplitude relationship (31) for the case of hinged-supported beam reads at the $\epsilon$-order:

$$
\Omega=1+\left[\frac{3}{16} \pi^{2}-\frac{\pi^{4}}{8}\left(\frac{1}{3}-\frac{3}{8 \pi^{2}}\right)\right] a^{2}
$$

The first term in the brackets accounts for the effect of the elastic term, the second one for the inertia term. In the relationship furnished by Atluri (1973) (equation (46)), omitting the rotary inertia term which gives negligible effects, the inertia term is the same while the elastic one is four times lower, notwithstanding the same approximations are introduced and the same nonlinear curvature is used as in the present work.

From the exact equilibrium equations for planar indeformable beam:

$$
\begin{gathered}
-(N \cos \theta)^{\prime}+(T \sin \theta)^{\prime}-m \ddot{u}=0 \\
-(N \sin \theta)^{\prime}-(T \cos \theta)^{\prime}+m \ddot{v}=p \\
-T-M^{\prime}=0
\end{gathered}
$$

the transversal equilibrium equation reads:

$$
\left(\frac{M^{\prime}}{\cos \theta}+\frac{\sin \theta}{\cos \theta} \int_{s}^{\ell} m \ddot{u} d s\right)^{\prime}+m \ddot{v}=p
$$

Since $u$ is a second-order variable, in the equation up to order-three it is correct to consider $\sin \theta / \cos \theta=\theta$ but in principle it cannot be assumed $\cos \theta=1$ in the first term, as followed in the paper by Atluri (1973). This is the reason why a different contribution of the elastic term is obtained here. 